\title{
A characterization of the Khavinson-Shapiro conjecture via Fischer operators
}

\author{
Hermann Render \\ School of Mathematics and Statistics, University College Dublin, \\ Belfield, Dublin 4, Ireland. Email: hermann.render@ucd.ie
}

\begin{abstract}
The Khavinson-Shapiro conjecture states that ellipsoids are the only bounded domains in euclidean space satisfying the following property $(\mathrm{KS})$ : the solution of the Dirichlet problem for polynomial data is polynomial. In this paper we show that property (KS) for a domain $\Omega$ is equivalent to the surjectivity of a Fischer operator associated to the domain $\Omega$.
\end{abstract}

\section{Introduction}

In the 19th century ellipsoidal harmonics have been used to prove that for any polynomial $p$ of degree $\leq m$ there exists a harmonic polynomial $h$ of degree $\leq m$ such that $h(\xi)=p(\xi)$ for all $\xi \in \partial E$ where $\partial E$ is the boundary of an ellipsoid $E$ in the euclidean space $\mathbb{R}^{d}$. It follows that an ellipsoid satisfies the following property defined for an arbitrary open subset $\Omega$ in $\mathbb{R}^{d}$ :

(KS) For any polynomial $p$ with real coefficients there exists a harmonic polynomial $h$ with real coefficients such that $h(\xi)=p(\xi)$ for all $\xi \in$ $\partial \Omega$.

The Khavinson-Shapiro conjecture [12] states that ellipsoids are the only bounded domains $\Omega$ in $\mathbb{R}^{d}$ with property (KS). Obviously a domain $\Omega$ has property (KS) if and only if the Dirichlet problem for polynomial data (restricted to the boundary) have polynomial solutions; for the Dirichlet problem we refer to [2] and [7]. The Khavinson-Shapiro conjecture has been confirmed for large classes of domains but it is still unproven in its full generality, and we refer the interested reader to the expositions [8], [17, [14, [10], [15], [11] and for further ramifications in [13] originating from the work [16].

\footnotetext{
${ }^{1} 2010$ Mathematics Subject Classification 31B05; 35J05. Keywords and phrases: Dirichlet problem, harmonic extension, Khavinson-Shapiro conjecture.
} 
In this paper we want to characterize the property (KS) by using Fischer operators. In our context we shall mean by a Fischer operator 2 an operator of the form

$$
F_{\psi}(q):=\Delta(\psi q) \text { for } q \in \mathbb{R}[x]
$$

where $\Delta$ is the Laplace operator $\frac{\partial^{2}}{\partial x_{1}^{2}}+\ldots .+\frac{\partial^{2}}{\partial x_{d}^{2}}$ and $\psi$ is a fixed element in $\mathbb{R}[x]$, the set of all polynomials in $d$ variables with real coefficients. Fischer operators often allows elementary and short proofs of mathematical statements which usually require hard and deep analysis, see [20], [11]. For example, the statement that ellipsoids have property (KS) can be proven in a few lines using Fischer operators and elementary results in Linear Algebra, see [4, [5], 3], and for further generalizations see [1], 12].

In order to formulate our main result we need some technical definitions. The zero-set of a polynomial $f \in \mathbb{R}[x]$ is denoted by $Z(f)=$ $\left\{x \in \mathbb{R}^{d}: f(x)=0\right\}$. We say that a subset $Z$ of $\mathbb{R}^{d}$ is an admissible common zero set if there exist non-constant irreducible polynomials $f, g \in \mathbb{R}[x]$ such that (i) $f \neq \lambda g$ for all $\lambda \in \mathbb{R}$ and (ii) $Z=Z(f) \cap Z(g)$. For dimension $d=2$ it is well known that an admissible common zero set is finite, see [19, p. 2]. For arbitrary dimension $d$ it is intuitively clear that an admissible common zero set has "dimension" $\leq d-2$ at each point.

We say that an open set $\Omega$ in $\mathbb{R}^{d}$ is admissible if for any $x \in \partial \Omega$, any open neighborhood $V$ of $x$ and for any finite family of admissible common zero sets $Z_{1}, \ldots, Z_{r}$ the set

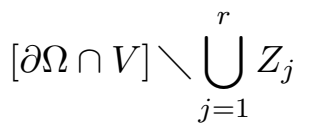

is non-empty. For dimension $d=2$ it is easy to see that an open set $\Omega$ is admissible if each point $x \in \partial \Omega$ is not isolated in $\partial \Omega$. For arbitrary dimension it seems to be difficult to formulate a precise topological condition but it is intuitively clear that a domain $\Omega$ is admissible if each point in the boundary $\partial \Omega$ has a neighborhood of dimension $d-1$.

The following is now the main result of this paper:

Theorem 1 Let $\Omega$ be an open admissible subset of $\mathbb{R}^{d}$. Then property (KS) holds for $\Omega$ if and only if there exists a non-constant polynomial $\psi \in \mathbb{R}[x]$ such that (i) $\partial \Omega \subset \psi^{-1}\{0\}$ and (ii) the Fischer operator $F_{\psi}: \mathbb{R}[x] \rightarrow \mathbb{R}[x]$ defined by $F_{\psi}(q):=\Delta(\psi q)$ for $q \in \mathbb{R}[x]$ is surjective.

An immediate consequence of Theorem 1 is that the Khavinson-Shapiro conjecture is true for all admissible bounded domains if the following purely

\footnotetext{
${ }^{2}$ More generaly one can define a Fischer operator by $q \longmapsto P(D)(\psi q)$ where $P(D)$ is a linear partial differential operator with constant real coefficients. Fischer's Theorem in $[6]$ states that the Fischer operator is a bijection whenever $\psi(x)$ is a homogeneous polynomial equal to the polynomial $P(x)$.
} 
algebraic conjecture of M. Chamberland and D. Siegel formulated in [5] is true:

(CS) The surjectivity of the Fischer operator $F_{\psi}: \mathbb{R}[x] \rightarrow \mathbb{R}[x]$ implies that the degree of $\psi$ is $\leq 2$.

We refer to [15] and [18] for more details on conjecture (CS) and related results. It should be emphasized that the polynomial $\psi$ in conjecture (CS) has real coefficients. In [9] it is shown for dimension $d=3$ that for any nonconstant polynomial $\varphi(z)=a_{0}+a_{1} z+\cdots+a_{n} z^{n}$ in the complex variable $z$ the operator $F_{\psi}: \mathbb{C}[x] \rightarrow \mathbb{C}[x]$ defined by

$$
F_{\psi}(q)=\Delta\left[\left(x_{3}-\varphi\left(x_{1}+i x_{2}\right)\right)^{2} q\left(x_{1}, x_{2}, x_{3}\right)\right]
$$

for $q \in \mathbb{C}[x]$ is surjective where $\mathbb{C}[x]$ is the set of all polynomials with complex coefficients.

\section{Proof of Theorem 1}

From [15] we cite the following result which is known as the Fischer decomposition of a polynomial.

Proposition 2 Suppose $\psi$ is a polynomial. Then the operator $F_{\psi}(q):=$ $\Delta(\psi q)$ is surjective if and only if every polynomial $f$ can be decomposed as $f=\psi q_{f}+h_{f}$, where $q_{f}$ is a polynomial and $h_{f}$ is harmonic polynomial

Proof of Theorem 1: The sufficiency is easy: By assumption, there exists $\psi \in \mathbb{R}[x]$ with $\partial \Omega \subset \psi^{-1}\{0\}$ such that $F_{\psi}$ is surjective. According to Proposition 2 there exists for each polynomial $f$ a polynomial $q$ and a harmonic polynomial $u$ such that $f=\psi q+u$. For $\xi \in \partial \Omega \subset \psi^{-1}\{0\}$ it follows that $f(\xi)=u(\xi)$. Thus $u$ is a polynomial solution to the Dirichlet problem of the domain $\Omega$ and property (KS) is satisfied.

Now assume that (KS) holds. Then there exists a harmonic polynomial $u \in \mathbb{R}[x]$ such that $|\xi|^{2}=u(\xi)$ for all $\xi \in \partial \Omega$. Define the polynomial $Q(x)=|x|^{2}-u(x)$. Then

$$
\partial \Omega \subset Q^{-1}(0)
$$

and $Q$ is a non-constant polynomial of degree $\geq 2$ since $\Delta(Q) \neq 0$. We factorize $Q(x)$ in irreducible factors, so

$$
Q(x)=|x|^{2}-u(x)=f_{1}^{m_{1}} \ldots f_{r}^{m_{r}}
$$

where $f_{k}$ is not a scalar multiple of $f_{l}$ for $k \neq l$, and $m_{k} \geq 1$ is the multiplicity of $f_{k}$. It follows that

$$
\partial \Omega \subset \bigcup_{k=1}^{r} f_{k}^{-1}(0) .
$$


Then $Z\left(f_{k}\right) \cap Z\left(f_{l}\right)$ is an admissible common zero set for $k \neq l$. Let $Z$ be the (finite) union of the sets $Z\left(f_{k}\right) \cap Z\left(f_{l}\right)$ with $k \neq l$. As $\Omega$ is admissible there exists $x \in \partial \Omega \backslash Z$, and now (2) implies that

$$
I:=\left\{k \in\{1, \ldots r\}: \exists x \in \partial \Omega \backslash Z \text { with } f_{k}(x)=0\right\}
$$

is non-empty. Let us define

$$
\psi:=\prod_{k \in I} f_{k}(x)
$$

We want to show that $F_{\psi}$ is surjective. By Proposition 2 it suffices to show that for any polynomial $f$ there is a harmonic polynomial $u$ and a polynomial $q$ such that $f=\psi q+u$. By property (KS) there exists a harmonic polynomial $u$ such that $f(\xi)=u(\xi)$ for all $\xi \in \partial \Omega$. Define $g(x)=f(x)-u(x)$, so $g$ vanishes on $\partial \Omega$. If we can show that each $f_{k}$ with $k \in I$ divides $g$ then, by irreducibility of $f_{k}$ and the condition that $f_{k} \neq \lambda f_{j}$ for $k \neq j$, we infer that $\psi=\prod_{k \in I} f_{k}$ divides $g$, say $g=\psi q$ for some polynomial $q$. Then $f-u=g=q \psi$ and we are done.

Let $k \in I$ be fixed and $g$ as above. Then there exists $x \in \partial \Omega \backslash Z$ with $f_{k}(x)=0$. Then $f_{l}(x) \neq 0$ for all $l \neq k$ since otherwise $x$ would be in $Z$. By continuity there is an open neighborhood $V$ of $x$ such that $f_{l}(y) \neq 0$ for all $y \in V$ and $l \neq k$. Then we conclude from (2) that

$$
\partial \Omega \cap V \subset f_{k}^{-1}(0) .
$$

Let us write $g=g_{1}^{m_{1}} \cdots g_{s}^{m_{r}}$ where $g_{1}, \ldots, g_{s}$ are irreducible polynomials such that $g_{j} \neq \lambda g_{l}$ for $j \neq l$. If $f_{k}=\lambda g_{j}$ for some $j \in\{1, \ldots, s\}$ we see that $f_{k}$ divides $g$. Assume that this is not the case and define $Z_{k}$ as the (finite) union of the admissible sets $Z\left(g_{j}\right) \cap Z\left(f_{k}\right)$ for $j=1, \ldots s$. Since $\Omega$ is admissible there exists $y \in \partial \Omega \cap V \backslash\left(Z \cup Z_{k}\right)$. The inclusion (3) shows that $f_{k}(y)=0$, and since $g$ vanishes on $\partial \Omega$ there exists $j \in\{1, . ., s\}$ such that $g_{j}(y)=0$. Hence $y \in Z\left(g_{j}\right) \cap Z\left(f_{k}\right) \subset Z_{k}$. Now we obtain a contradiction since $y \in \partial \Omega \cap V \backslash\left(Z \cup Z_{k}\right)$.

It remains to prove that $\partial \Omega$ is contained in $\psi^{-1}(0)$. If $j \in\{1, \ldots r\} \backslash I$ then it follows from the definition of $I$ that $f_{j}(x) \neq 0$ for all $x \in \partial \Omega \backslash Z$. This fact and (2) imply that

$$
\partial \Omega \backslash Z \subset \bigcup_{k \in I} f_{k}^{-1}(0)=: F .
$$

Let $x \in \partial \Omega$. Since $\Omega$ is admissible there exists for any ball $V$ with center $x$ and radius $1 / m$ an element $x_{m} \in(\partial \Omega \cap V) \backslash Z$. Then (4) shows that $x_{m} \in F$. Since $x_{m}$ converges to $x$ and $F$ is closed we infer that $x \in F$. Thus $\partial \Omega \subset F$. 


\section{References}

[1] D. H. Armitage, The Dirichlet problem when the boundary function is entire, J. Math. Anal. Appl. 291 (2004), 565-577.

[2] D. H. Armitage, S. J. Gardiner, Classical Potential Theory, Springer, London 2001.

[3] S. Axler, P. Gorkin, K. Voss, The Dirichlet problem on quadratic surfaces, Math. Comp. 73 (2003), 637-651.

[4] J. A. Baker, The Dirichlet problem for ellipsoids, Amer. Math. Monthly, 106 (1999), 829-834.

[5] M. Chamberland, D. Siegel, Polynomial solutions to Dirichlet problems, Proc. Amer. Math. Soc.v129 (2001), 211-217.

[6] E. Fischer, Über die Differentiationsprozesse der Algebra, J. für Math. (Crelle Journal) 148 (1917), 1-78.

[7] S.J. Gardiner, The Dirichlet problem with non-compact boundary, Math. Z. 213 (1993), 163-170.

[8] L. Hansen, H.S. Shapiro, Functional Equations and Harmonic Extensions, Complex Var. 24 (1994), 121-129.

[9] D. Khavinson, Singularities of harmonic functions in $\mathbb{C}^{n}$, Proc. Symp. Pure Applied Math., Amer. Math. Soc., Providence, RI, 52 (1991), Part $3,207-217$.

[10] D. Khavinson, E. Lundberg, The search for singularities of solutions to the Dirichlet problem: recent developments, Hilbert spaces of analytic functions, 121-132, CRM Proc. Lecture Notes, 51, Amer. Math. Soc., Providence, RI, 2010.

[11] D. Khavinson, E. Lundberg, A tale of ellipsoids in Potential Theory, Notices Amer. Math. Soc. 61 (2014), 148-156.

[12] D. Khavinson, H. S. Shapiro, Dirichlet's Problem when the data is an entire function, Bull. London Math. Soc. 24 (1992), 456-468.

[13] D. Khavinson, N. Stylianopoulos, Recurrence relations for orthogonal polynomials and algebraicity of solutions of the Dirichlet problem, "Around the Research of Vladimir Maz'ya II, Partial Differential Equations", 219-228, Springer, 2010.

[14] E. Lundberg, Dirichlet's problem and complex lightning bolts, Comp. Meth. Funct. Theory, 9 (2009), 111-125. 
[15] E. Lundberg, H. Render, The Khavinson-Shapiro conjecture and polynomial decompositions, J. Math. Anal. Appl. (2011), 506-513.

[16] M. Putinar, N. Stylianopoulos, Finite-term relations for planar orthogonal polynomials, Complex Anal. Oper. Theory 1 (2007), 447-456.

[17] H. Render, Real Bargmann spaces, Fischer decompositions and sets of uniqueness for polyharmonic functions, Duke Math. J. 142 (2008), 313352.

[18] H. Render, The Khavinson-Shapiro conjecture for domains with a boundary consisting of algebraic hypersurfaces, submitted.

[19] I.R. Shafarevich, Basic Algebraic Geometry, Springer 1994, 2nd edition,

[20] H.S. Shapiro, An algebraic theorem of E. Fischer and the Holomorphic Goursat Problem, Bull. London Math. Soc. 21 (1989), 513-537. 